\title{
BER Performance of PDM 4-QAM Optical Transmission System Considering the Effects of PMD and GVD Using Exact Probability Density Function
}

\author{
Kazi Abu Taher ${ }^{1}$, Satya Prasad Majumder ${ }^{2}$ \\ ${ }^{1}$ Department of Electrical and Electronic Engineering, American International University Bangladesh, Dhaka, Bangladesh \\ ${ }^{2}$ Department of Electrical and Electronic Engineering, Bangladesh University of Engineering and Technology, Dhaka, Bangladesh
}

Email address:

kataher@yahoo.com (K. A. Taher)

To cite this article:

Kazi Abu Taher, Satya Prasad Majumder. BER Performance of PDM 4-QAM Optical Transmission System Considering the Effects of PMD and GVD Using Exact Probability Density Function. American Journal of Optics and Photonics. Vol. 6, No. 1, 2018, pp. 14-19.

doi: 10.11648/j.ajop.20180601.13

Received: January 20, 2018; Accepted: February 11, 2018; Published: March 14, 2018

\begin{abstract}
A noble theoretical approach is presented to evaluate the bit error rate (BER) performance of an optical polarization division multiplexed (PDM) 4-multilevel quadrature amplitude modulation (4-QAM) transmission system under the combined influence of polarization mode dispersion (PMD) and group velocity dispersion (GVD) in a single mode fiber (SMF). The analysis is carried out considering a coherent homodyne receiver. The exact probability density function (pdf) fluctuation due to PMD and GVD is evaluated from its moments using a Monte-Carlo simulation technique. Average BER is evaluated by averaging the conditional BER over the pdf of the random phase fluctuation. BER performance results are evaluated for different system parameters. It is found that PDM 4-QAM coherent homodyne system doubles the data rate but suffers more power penalty than the 4-QAM system. Results show that for a BER of $10^{-9}$ at DGD of $0.5 \mathrm{~T}$ and GVD value of $1700 \mathrm{ps} / \mathrm{nm}$ the PDM 4-QAM system needs $2.21 \mathrm{~dB}$ more power than 4-QAM systems giving the leverage of doubling the data rate.
\end{abstract}

Keywords: Quadrature Amplitude Modulation, Polarization Division Multiplexing, Polarization Mode Dispersion, Group Velocity Dispersion

\section{Introduction}

Ever increasing data rates, temperature changes, power variations and changes in stresses induce birefringence causing GVD and PMD values of the optical fiber to the levels that exceed the system tolerances [1-2]. The impacts of PMD and GVD in SMF are of great interests in current and next generation high speed optical data transmission systems [3, 4]. The latest drive of coherent optical data transmission system augmented by high speed and efficient digital signal processing (DSP) could contribute to the spectral efficiency (SE). The technique of PDM is used with QAM modulation systems to achieve high transmission rate through the legacy network which is designed to handle only one fourth of the transmission bandwidth. Further polarization diversity coherent receiver is proved to be a promising technology for high data transmission networks. Higher level modulation formats increased the SE but the tolerance of the system to
GVD and PMD are even getting smaller. Again, optical homodyne coherent transmission system provides a viable approach in increasing SE with higher sensitivity [5, 6]. The limitations imposed by GVD and PMD are very important for the reliable high speed data transmission.

In long haul single mode fiber (SMF), main part of the PMD effect comes from frequency independent first order PMD that is the differential group delay (DGD). Higher order PMD effects may be neglected. For conventional detection systems, the impairments induced by a constant DGD scales with the square of the bit rate, resulting in drastic PMD induced degradation in high speed transmission systems [7, 8]. At higher data rate, the effects of PMD are difficult to analyze due its stochastic nature. Further, the random fluctuations of the fiber birefringence affect the correlation length and the beat length of the fiber $[9,10]$. Use of exact pdf approach gives a realistic picture of the combined effect of PDM and DGD [1]. 
In this paper, a noble analytical approach is presented to evaluate the exact pdf of the phase fluctuation due to combined effect of PMD and GVD which is then used to evaluate the system BER. Exact pdf of the phase fluctuation is evaluated analytically as well as by Monte Carlo simulation. BER is then evaluated for different system parameters. Power penalty suffered by the system is also evaluated. This paper is organized as follows; system model is discussed in Section 2. Section 3 covers the theoretical analysis of BER with GVD and PMD effects in a m-QAM system without and with PDM. Section 4 covers the results and discussions and Section 5 provides the conclusion.

\section{System Model}

Figure 1 depicts the block diagram of an optical PDM 4-QAM transmission system showing the transmitter section, the SMF link with EDFA and polarization diversity coherent receiver. The transmitter section as shown in Figure 1(a), consists of two QAM modulators one for each of the two polarizations. Each polarization modulates $\mathrm{k}=\log _{2}(\mathrm{M})$, where $\mathrm{M}$ is the alphabet size, data streams. The input laser is passed to a polarization beam splitter (PBS) to split the laser into two polarizations $\mathrm{E}_{\mathrm{x}}$ and $\mathrm{E}_{\mathrm{y}}$. One of these polarized lasers is passed to one of the two QAM modulators which are used as the carrier to modulate the QAM signals. When single polarization is used,
4-QAM takes 2 data streams $\mathrm{D}_{1}$ and $\mathrm{D}_{2}$ as input, but when both polarizations are used, PDM 4-QAM takes four data streams $\mathrm{D}_{\mathrm{x}, 1}, \mathrm{D}_{\mathrm{x}, 2}, \mathrm{D}_{\mathrm{y}, 1}$ and $\mathrm{D}_{\mathrm{y}, 2}$ as input where $\mathrm{x}$ and $\mathrm{y}$ denote the $\mathrm{x}$ and $\mathrm{y}$ polarizations respectively. In case of 16-QAM system, there are four data streams given as the input of the QAM modulator. But in case of PDM 16-QAM system there are eight input data streams. Outputs of the two QAM modulators are combined at the polarization beam combiner (PBC) to form the transmitter signal. The fiber link consists of SMF sections each of $100 \mathrm{~km}$ with EDFA as shown in Figure 1(b).

The receiver block diagram of a PDM 4-QAM with polarization diversity optical transmission system is shown in Figure 1(c). The received signal $\mathrm{E}_{\mathrm{r}}(\mathrm{t})$ is fed to a PBS without any polarization controller (PC) in PDM systems. But in single polarization transmission, $\mathrm{PC}$ is used in place of PBS. One of the outputs of the PBS is X-polarized which is fed to the upper $90^{\circ}$ hybrid. Another output of the PBS is Y-polarized which is fed to the lower $90^{\circ}$ hybrid. For higher QAM modulation, the number of $90^{\circ}$ hybrid is increased proportionately. The output of the local oscillator (LO) laser is linearly polarized at $45^{\circ}$ with respect to the receiver polarization which is fed into the lower PBS. Outputs of the lower PBS are fed as the lower input of the $90^{\circ}$ hybrids as shown in Figure 1(c). The received signal is thus coupled to the LO laser in the $90^{\circ}$ hybrids to implement the homodyne detection.

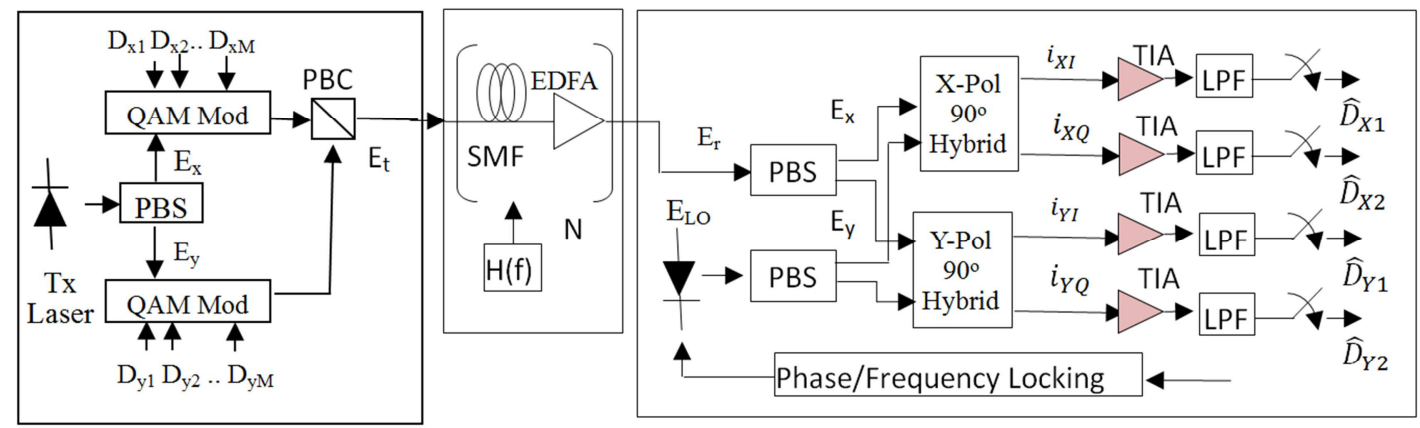

(a)

(b)

(c)

Figure 1. Block diagram of a PDM 4-QAM transmission system with (a) Transmitter (b) SMF link with EDFA and (c) Polarization diversity coherent receiver without DSP unit.

The 3-dB couplers of the $90^{\circ}$ hybrids beat the LO laser with the received signal. Each $90^{\circ}$ hybrid passes the beat signals to the photo detectors (PDs) for conversion from optical domain to electrical domain using balanced detection. The PDs detect only the difference signals to generate the baseband signals using the homodyne approach. The down-converted baseband signals are fed into the trans-impedance amplifiers (TIA) for converting them to voltages. The output of the TIA are passed through the LPF and then through the comparator for detection of the signals $\widehat{D}_{\mathrm{X}, 1}, \widehat{D}_{\mathrm{X}, 2}, \widehat{D}_{\mathrm{Y}, 1}$ and $\widehat{D}_{\mathrm{Y}, 2}$. In coherent detection, this electrical data is further processed in the digital signal processing (DSP) unit which is beyond the scope of this paper. $\mathrm{PC}$ is required to align the principal states of polarization (PSP) to that of the receiver. But the polarization diversity receiver as shown in Figure 1(c) does not require any PC.

\section{Theoretical Analysis}

An optical PDM m-QAM signal hosts two m-QAM signals, one m-QAM modulated signal in each polarization. Again each $\mathrm{m}$-QAM signal has $\mathrm{k}=\log _{2}(\mathrm{M})$ data streams, where $\mathrm{M}$ is the number of alphabet size. A 4-array quadrature amplitude modulation (4-QAM) modulator processes two data streams. PDM-4-QAM system processes four data streams. A 16-QAM with single polarization processes four data streams and PDM-16-QAM modulator processes eight data streams. To modulate the QAM signal, two orthogonal basis functions are used:

$$
\psi_{I}(t)=\sqrt{\frac{2}{T}} \cos \left(2 \pi f_{c} t\right), 0 \leq t \leq T
$$




$$
\psi_{Q}(t)=\sqrt{\frac{2}{T}} \sin \left(2 \pi f_{c} t\right), 0 \leq t \leq T
$$

where $f_{c}$ and $T$ denotes the carrier frequency and the symbol duration respectively. The transmitter output signal is given by $E_{t}(\mathrm{t})=\left[\begin{array}{l}E_{t, x}(\mathrm{t}) \\ E_{t, y}(\mathrm{t})\end{array}\right]$, where the two orthogonal polarization signal components are given by [1]:

$$
\begin{aligned}
& E_{t, x}(\mathrm{t})=\sqrt{E_{0}} p(t) I_{x, i} \psi_{I, x}(t)-\sqrt{E_{0}} p(t) Q_{x, i} \psi_{Q, x}(t) \\
& E_{t, y}(\mathrm{t})=\sqrt{E_{0}} p(t) I_{y, i} \psi_{I, y}(t)-\sqrt{E_{0}} p(t) Q_{y, i} \psi_{Q, y}(t)
\end{aligned}
$$

where $\mathrm{i}=1,2, \ldots . \mathrm{M}, \mathrm{M}$ is the alphabet size, $I_{x, i}, I_{y, i}, Q_{x, i}$ and $Q_{y, i}$ represent are the amplitudes of the quadrature carriers amplitude modulated by the information symbols of $\mathrm{x}$ - and y-polarizations, and $p(t)$ is the pulse shape. The electric field at the output of the fiber is given by

$$
E_{r}(\mathrm{t})=E_{t, x}(\mathrm{t}) \otimes h_{1}(t)+E_{t, y}(\mathrm{t}) \otimes h_{2}(t)
$$

where $\otimes$ denotes convolution, $h_{1}(t)$ and $h_{2}(t)$ are the inverse Fourier transforms of the low-pass equivalent transfer function of a lossless fiber $H_{1}(f)$ and $H_{2}(f)$ respectively, which includes the effects of PMD and GVD. The transfer functions are given by [1]:

$$
\begin{aligned}
& H_{1}(f)=\sqrt{\alpha} \exp \left[j 2 \pi f\left(-\frac{\Delta \tau}{2}\right)-j \gamma(\pi f T)^{2}\right] \\
& H_{2}(f)=\sqrt{1-\alpha} \exp \left[j 2 \pi f\left(\frac{\Delta \tau}{2}\right)-j \gamma(\pi f T)^{2}\right]
\end{aligned}
$$

where $\alpha$ denotes the power splitting ratio between $x$ and $y$ polarizations, $\Delta \tau$ represent the DGD between the two PSPs, and $\gamma$ is the accumulated GVD of the fiber link. Here, we assume that there is a negligible amount of polarization dependent loss (PDL). Assuming linear phase approximation, lossless couplers and coherent homodyne detection, the signal at the outputs of the $\mathrm{x}$ - and y-polarized $90^{\circ}$ hybrid couplers are similar. Considering only the $\mathrm{x}$-polarized $90^{\circ}$ hybrid, the output after the balanced detectors is given by:

$$
\begin{aligned}
i_{X I}(t) & =P \cos \left(\frac{\pi \cdot \phi_{X}^{\prime \prime}}{2}\right)(t)\left[I_{X, i}, \cos \left(\phi_{X I}(t)\right)\right]+i_{n, X I} \\
i_{X Q}(t) & =P \cos \left(\frac{\pi \cdot \phi_{X}^{\prime \prime}}{2}\right)(t)\left[Q_{X, i} \cos \left(\phi_{X Q}(t)\right)\right]+i_{n, X Q}
\end{aligned}
$$

where $i_{n, X I}$ and $i_{n, X Q}$ are the total noise components at the two quadrature that consist of shot noise and receiver thermal noise, $P=2 R_{d} \sqrt{P_{S} P_{L O}}, R_{d}$ is the responsivity of the photodetector, $P_{s}$ is the output power at the fiber receiving end, $\mathrm{P}_{\mathrm{LO}}$ is the local oscillator power, $I_{X, i}$ and $Q_{X, i}$ represent the amplitude modulated data bits, $\phi_{X I}$ and $\phi_{X Q}$ are the phase angles including the phase noise in the $\mathrm{x}$-polarized quadrature components, and $\cos \phi_{X}^{\prime \prime}(t)$ represents the amplitude of the random phase angle fluctuations due to DGD and GVD in $\mathrm{x}$-polarization as in [1]. The value of $\phi_{X}^{\prime \prime}(t)$ is calculated using the mean of the phases changes of the transmitted and received training data sequences. The phases $\phi_{X I}$ and $\phi_{X Q}$ are given by:

$$
\begin{aligned}
& \phi_{X I}(t)=\left(\frac{2 \pi}{M}\right) \sum_{k} g_{k I}(t-k T) \\
& \phi_{X Q}(t)=\left(\frac{2 \pi}{M}\right) \sum_{k} g_{k Q}(t-k T)
\end{aligned}
$$

where $\mathrm{k}$ is the number of samples for each symbol, $g(t)=$ $\operatorname{Re}\left[p(t) \otimes h_{1}(t)\right]$ represents the pulse shape of the received signal and $M$ is the alphabet size. The baseband signal currents at the output of the balanced detector are:

$$
\begin{aligned}
& i_{X I}(t)=P I_{X, i} \cos \phi_{X}^{\prime \prime}(t) \cos \hat{\phi}_{X I}(t)+i_{n, X I}(t) \\
& i_{X Q}(t)=P Q_{X, i} \cos \phi_{X}^{\prime \prime}(t) \sin \hat{\phi}_{X Q}(t)+i_{n, X Q}(t)
\end{aligned}
$$

where and, $i_{n, x, I}(t)$ and $i_{n, x, Q}(t)$ are the receiver noise with variance $\sigma_{n}^{2}$ details of which is given at [9]. Details of the shot noise, thermal noise and amplified spontaneous emission (ASE):

$$
\sigma_{n}^{2}=\sigma_{\text {shot }}^{2}+\sigma_{\text {thermal }}^{2}+\sigma_{\text {ASE }}^{2}
$$

where $\sigma_{\text {shot }}^{2} \cong 2 \mathrm{qR}_{\mathrm{C} 1} \mathrm{P}_{\mathrm{LO}} \mathrm{B}\left[1+\delta-2 \sqrt{(1-\mu) \delta_{\mathrm{X}}} \cos \emptyset_{\mathrm{X}, \mathrm{I}}\right]+$ $\left.2 q \mathrm{R}_{\mathrm{C} 2} \mathrm{P}_{L O} \mathrm{~B}\left[1+\delta+2 \sqrt{(1-\mu) \delta} \cos \emptyset_{X, I}\right)\right]$

$$
\begin{aligned}
& \sigma_{\text {thermal }}^{2}=2\left(1-r_{t h}\right) i_{t h}^{2} \mathrm{~B} \\
& \sigma_{\text {ASE }}^{2}=\sigma_{\text {ASE,shot }}^{2}+\sigma_{\mathrm{s}-\mathrm{sp}}^{2}+\sigma_{\mathrm{sp}-\mathrm{sp}}^{2}
\end{aligned}
$$

$$
\begin{gathered}
=2 q \eta B\left(I_{i} G+I_{s p}\right)+4 G \eta^{2} I_{i} I_{s p} \frac{B}{B_{o}}+\eta^{2} I_{s p}^{2}\left(2-\frac{B}{B_{o}}\right) \frac{B}{B_{o}}, 0 \\
<B \leq \frac{B_{o}}{2}
\end{gathered}
$$

where $\mathrm{q}$ is the electric charge, $\mathrm{R}_{\mathrm{C} 1}$ and $\mathrm{R}_{\mathrm{C} 2}$ are the effective responsivity of the PDs at the port $\mathrm{C}_{1}$ and $\mathrm{C}_{2}$ of $90^{\circ}$ hybrid, $\mathrm{P}_{\mathrm{LO}}$ is the LO power, $B$ is the data bandwidth, $\delta$ is the ratio of signal power to the LO power, $\mu$ is the quadrature power imbalance ratio, and $\emptyset_{\mathrm{X}, \mathrm{I}}$ is the phase angle of the $\mathrm{x}$-polarized in-phase signal considering no correlation coefficient between single-ended noise current densities. Here, $r_{t h}$ is the correlation coefficient of the differential thermal noise power of identical single ended thermal noise sources, $i_{\text {th }}$ is the input equivalent noise current spectral density of the single-ended input, $\eta$ is the quantum efficiency, $I_{i}$ is the photocurrent equivalent of the signal power, $I_{s p}$ is the photocurrent equivalent of the ASE noise power, $G$ is the gain of the amplifier and $\mathrm{B}_{\mathrm{o}}$ is the optical bandwidth.

The output of the TIA excluding the noise is given by,

$$
\begin{array}{r}
v_{X I, i}(t)=A_{I, i}(t) \cos \left(\Delta \phi_{o, X I}(t, \theta)\right) \\
v_{X Q, i}(t)=A_{Q, i}(t) \cos \left(\Delta \phi_{o, X Q}(t, \theta)\right)
\end{array}
$$

where $\Delta \phi_{o, X I}(t, \theta)$ and $\Delta \phi_{o, X Q}(t, \theta)$ are the differential output phases jointly influenced by $\Delta \tau$ and $\gamma$, and

$$
\begin{gathered}
A_{I, i}(t)=2 R_{d} \sqrt{P_{S} P_{L O}} I_{x, i} \cos \left(\frac{\pi \cdot \phi_{X}^{\prime \prime}}{2} t\right) \\
A_{Q, i}(t)=2 R_{d} \sqrt{P_{S} P_{L O}} Q_{x, i} \cos \left(\frac{\pi \cdot \phi_{X}^{\prime \prime}}{2} t\right)
\end{gathered}
$$


Then, we define the signal-noise-ratio (SNR) as:

$$
\operatorname{SNR}(\theta)=A(\theta) /\left(2{\sigma_{n}}^{2}\right)
$$

where $A_{i}(\theta)=v_{X I, i}{ }^{2}+v_{X Q, i}{ }^{2}$ is the output power at the output of the TIA. The conditional BER for the phase modulated signal is:

$$
\operatorname{BER}(\theta)=0.5 * \operatorname{erc} f \sqrt{\operatorname{SNR}(\theta)}
$$

When the impact of cross-polarization is considered, the crosstalk-term is given by [9]:

$$
\begin{array}{r}
\mathrm{i}_{\mathrm{Xtalk}, \mathrm{C}}=\varepsilon_{\mathrm{X}}^{2}\left[\mathrm{R}_{\mathrm{C} 2}-\mathrm{R}_{\mathrm{C} 1}\right] \mathrm{P}_{\mathrm{Y}}+ \\
2 \varepsilon_{\mathrm{X}}\left[\mathrm{R}_{\mathrm{C} 2}+\mathrm{R}_{\mathrm{C} 1}\right] \sqrt{\mathrm{P}_{\mathrm{Y}, \mathrm{I}} \mathrm{P}_{\mathrm{LO}, \mathrm{X}}} \cos \phi_{\mathrm{Y}, \mathrm{I}}+2 \varepsilon_{\mathrm{X}} \varepsilon_{\mathrm{F}}\left[\mathrm{R}_{\mathrm{C} 2}+\right. \\
\left.\mathrm{R}_{\mathrm{C} 1}\right] \sqrt{\frac{\kappa}{2}} \mathrm{P}_{\mathrm{X}, \mathrm{I}} \sqrt{\frac{\kappa}{2}} \mathrm{P}_{\mathrm{Y}, \mathrm{I}} \cos \left(\phi_{\mathrm{X}, \mathrm{I}}-\phi_{\mathrm{Y}, \mathrm{I}}\right)
\end{array}
$$

where $i_{X t a l k, C}$ is the crosstalk current due to co-propagating sub-channel of the PDM system at port $\mathrm{C}$ of the $90^{\circ}$ hybrid. $\varepsilon_{\mathrm{F}}$ and $\varepsilon_{\mathrm{X}}$ are the complex fading and crosstalk coefficients respectively, $\mathrm{P}_{Y}, \mathrm{P}_{\mathrm{Y}, \mathrm{I}} \mathrm{P}_{\mathrm{X}, \mathrm{I}}$ and $\mathrm{P}_{\mathrm{LO}, \mathrm{X}}$ are the received signal power at Y-polarization, in-phase of Y-polarization, in-phase of X-polarization and portion of LO power at X-polarization respectively, $\kappa$ is the transformation constant, $\phi_{X, I}$ is the phase angle of the $\mathrm{X}$-polarization signal and $\phi_{Y, I}$ is the phase angle of the Y-polarization signal. When cross-polarization is considered, $\operatorname{SNR}(\theta)$ will change to signal to crosstalk plus noise ratio $\operatorname{SCNR}(\theta)$, given by [9]:

$$
\operatorname{SCNR}(\theta)=\mathrm{V}^{2}(\theta) /\left[2 \sigma_{\mathrm{n}}{ }^{2}+\left(\mathrm{i}_{\mathrm{Xtalk}, \mathrm{C}}\right)^{2}\right]
$$

The calculated conditional BER is given by:

$$
\operatorname{BER}(\theta)=0.5 * \operatorname{erc} f \sqrt{\operatorname{SCNR}(\theta)}
$$

Then the average BER can be found out by knowing the pdf of random phase $\theta$ that represents the random phase fluctuations due to DGD and GVD. The pdf of $\theta$ can be obtained from the exact pdf calculated using the training data. The exact pdf of the randon phase is determined from the moments of the training data sequences that gives the characteristic function and thereafter by inverting the characteristic function of the phase angle. Definition of the characteristic function of random phase $\theta$ is given as [1],

$$
F_{\theta}(j \theta)=\sum_{n=1}^{\infty} \frac{(j \theta)^{2 n}}{(2 n) !} M_{2 n}
$$

where $M_{2 n}$ is the even order moments of the random output phase $\theta$ of the received training data sequence which is under the combined affect of GVD and PMD. Moments $\mathrm{M}_{2 \mathrm{n}}$ can be determined by using the following relation of (24). Exact pdf of output random phase can be calculated taking inverse Fourier transform as:

$$
f_{\theta}(\theta)=F^{-1}\left(F_{\theta}(j \theta)\right)
$$

Then, the average BER can be obtained as:

$$
\operatorname{BER}=\int_{-\infty}^{\infty} \operatorname{BER}(\theta) f_{\theta}(\theta) \mathrm{d} \theta
$$

\section{Results and Discussion}

Following the analytical approach developed above, we evaluate the BER from the conditional BER, considering the phase fluctuation due to only GVD or PMD in 4-QAM and PDM 4-QAM systems. To evaluate the effect of DGD, the GVD is considered to be zero and to evaluate the effect of the GVD, the DGD is set zero in (6) and (7). In a PDM system, both the polarizations carry data independently. Thus the impact of transfer function on each polarization is also unaffected by the transfer function other sub-channel. With this, we investigate the impact of any one polarization. The statistical properties of PMD have been experimentally and theoretically studied. It was founded that the main effects of PMD comes from DGD and the impact from the higher order frequency dependent PMD terms may be neglected. The BER and power penalty are found out considering the presence of both PMD and GVD. This paper considers the data rate of $40 \mathrm{Gbps}$ in each polarization throughout unless specified otherwise.

Table 1. Different parameters and their values.

\begin{tabular}{ll}
\hline Parameter & Value \\
\hline Over sampling factor & 20 \\
Duration of TX/RX-filters (symbols) & 30 \\
CD of the SMF & $17 \mathrm{ps} / \mathrm{nm}-\mathrm{km}$ \\
Effective responsivity at port C1 & $0.20 \mathrm{~A} / \mathrm{W}$ \\
Effective responsivity at port C2 & $0.24 \mathrm{~A} / \mathrm{W}$ \\
Phase of the X-polarized signal $\emptyset_{\mathrm{X}}$ & $45^{0}$ \\
Phase of the X-polarized signal $\emptyset_{\mathrm{X}}$ & $45^{0}$ \\
Power split ratio between polarizations & 0.42 \\
Local oscillator power split ratio & 0.45 \\
SMF link length & $100 \mathrm{~km}$ \\
Attenuation loss & $0.2 \mathrm{~dB} / \mathrm{km}$ \\
Optical carrier wavelength & $1550 \mathrm{~nm}$ \\
Spontaneous emission factor & 1.4 \\
Gain of the EDFA & $20 \mathrm{~dB}$ \\
Optical filter bandwidth & $100 \mathrm{GHz}$ \\
Local oscillator power output & $7 \mathrm{dBm}$ \\
Photocurrent equivalent of the signal, $\mathrm{I}_{\mathrm{i}}$ & $15.51 \mu \mathrm{A}$ \\
Photocurrent equivalent of ASE noise, $\mathrm{I}_{\mathrm{sp}}$ & $4.21 \mu \mathrm{A}$ \\
Input equivalent noise current density, $\mathrm{n}$ th & $15 \mathrm{e}-12 \mathrm{~A} / \mathrm{Hz}$ \\
\hline
\end{tabular}

Figure 2 shows the exact pdf for the distribution of the phase fluctuation due to GVD for fiber link lengths of 100 , 300 and $500 \mathrm{~km}$ with GVD values of 1700,5100 and 8500 $\mathrm{ps} / \mathrm{nm}$ by using (25).

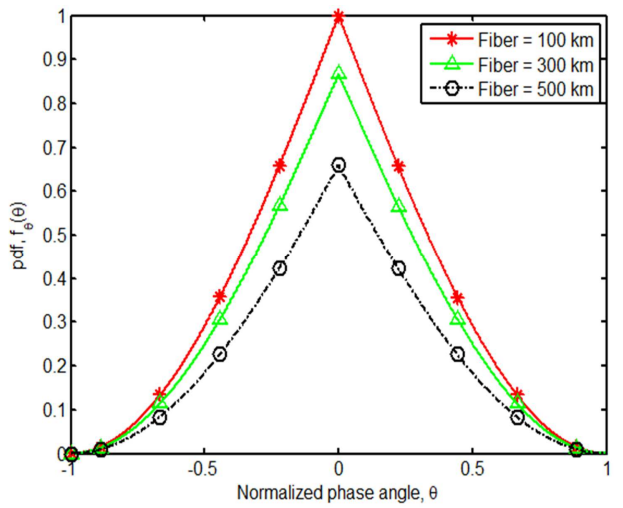

Figure 2. Pdf of exact distribution for link lengths of 100, 300 and $500 \mathrm{~km}$ corresponding to GVD values of 1700,5100 and $8500 \mathrm{ps} / \mathrm{nm}$ respectively. 
Figure 3 shows the BER as a function of received signal power for link lengths of 0,300 and $500 \mathrm{~km}$ corresponding to GVD values of 0,5100 and $8500 \mathrm{ps} / \mathrm{nm}$ respectively with DGD value of zero without and with PDM. The graph shows the effect of effect of GVD on the BER in the absence of PMD and considering the presence of different values of GVD represented by distance using the exact distribution. Different BER plots for instantaneous differential phases caused by different values of GVD introduces different amount of phase noise and the noise increases with the increase of distance. The fiber length considered is 0,300 and $500 \mathrm{~km}$ corresponding to GVD values of 0,5100 and $8500 \mathrm{ps} / \mathrm{nm}$. It is clear that the PDM 4-QAM needs more power for achieving the same BER. At $500 \mathrm{~km}$ of fiber link length corresponding to GVD of $8500 \mathrm{ps} / \mathrm{ns}$ for achieving BER of $10^{-9}$, the PDM 4-QAM system needs $4.5 \mathrm{~dB}$ more signal power when compared with 4-QAM systems.

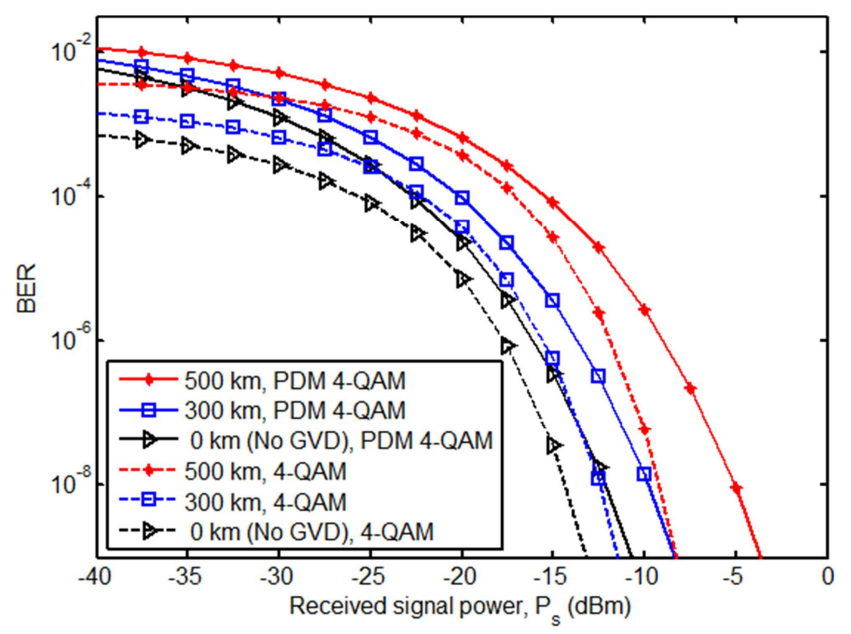

Figure 3. BER as a function of received signal power for link lengths of 0, 300 and $500 \mathrm{~km}$ corresponding to GVD values of 0,5100 and $8500 \mathrm{ps} / \mathrm{nm}$ respectively with DGD values of zero and without and with PDM.

Figure 4 shows the graphs of BER as a function of received optical signal power in the presence of DGD only in the absence of GVD, at DGD values of $0,0.3$ and $0.5 \mathrm{~T}_{\mathrm{b}}$ by using exact distribution of the phase fluctuation. At DGD of $0.5 \mathrm{~T}_{\mathrm{b}}$ and BER of $10^{-6}$, the PDM 4-QAM system needs required signal power is $-13.15 \mathrm{dBm}$ using exact pdf. But 4-QAM needs $15.0 \mathrm{dBm}$ received signal power for achieving same BER of $10^{-6}$ at DGD of $0.5 \mathrm{~T}_{\mathrm{b}}$. But at BER or $10^{-9}$ and DGD of $0.5 \mathrm{~T}_{\mathrm{b}}$, the requirements of the received signal powers are 2.5 $\mathrm{dBm}$ and $10.5 \mathrm{dBm}$ for PDM 4-QAM and 4-QAM systems respectively. Thus it is evident from the graphs that the PDM system needs more power than 4-QAM system but gives the advantage of doubling the data transmission rate.

Figure 5 shows the graph of required signal power, $\mathrm{P}_{\text {SIG }}$ as a function of normalized DGD for BER of $10^{-9}$. The graph shows required signal power as a function of normalized DGD for link lengths of 0,50 and $100 \mathrm{~km}$ corresponding to GVD values of 0,850 and $1700 \mathrm{ps} / \mathrm{nm}$ respectively without and with PDM. It is noted that for achieving the BER of $10^{-9}$, the requirement of the received power increases with the increase of DGD.

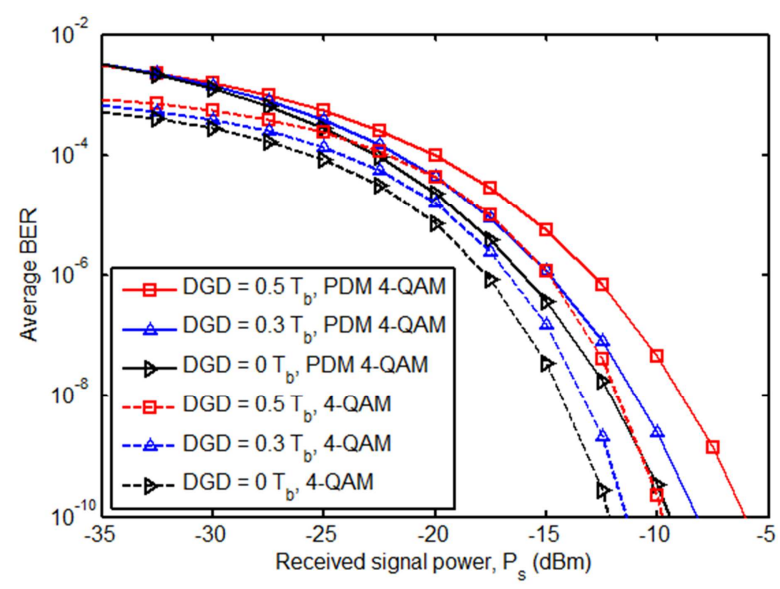

Figure 4. BER as a function of received signal power for DGD of 0.5T, 0.3T and 0 with GVD values of zero, without and with PDM.

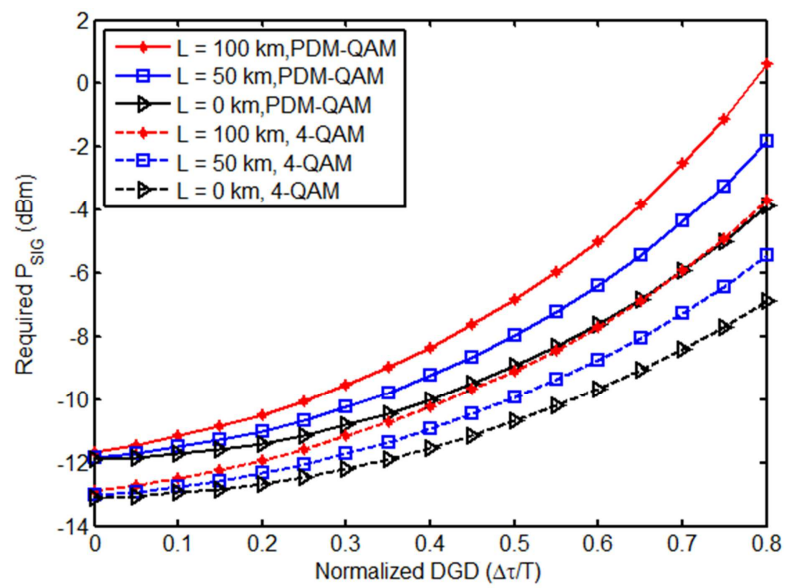

Figure 5. Required signal power as a function of normalized DGD for link lengths of 0, 50 and $100 \mathrm{~km}$ corresponding to GVD values of 0, 850 and 1700 $\mathrm{ps} / \mathrm{nm}$ respectively without and with PDM.

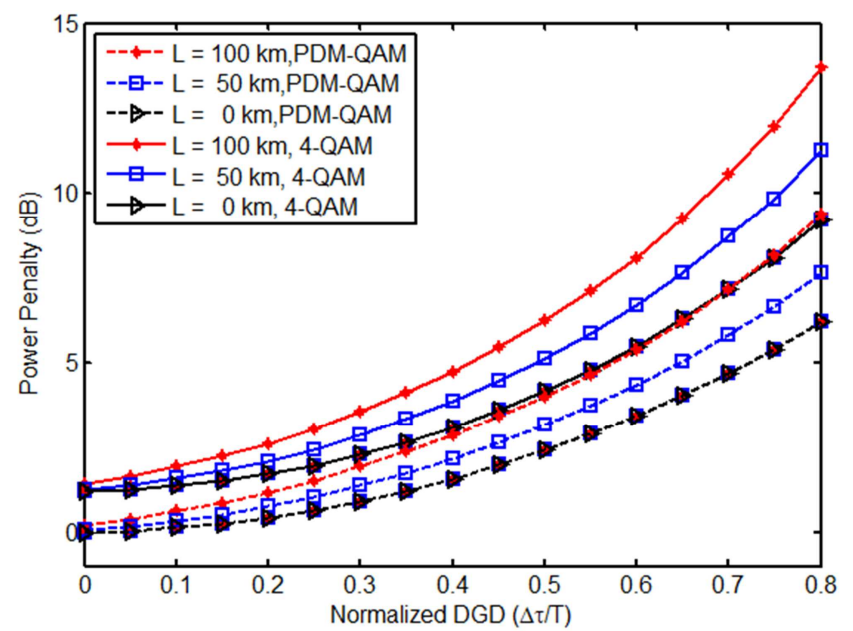

Figure 6. Power penalty as a function of normalized DGD for link lengths of 0 , 50 and $100 \mathrm{~km}$ corresponding to GVD values of 0,850 and $1700 \mathrm{ps} / \mathrm{nm}$ respectively without and with PDM taking the required signal power at 0 $D G D$ and $0 G V D$ as reference.

Figure 6 shows the graphs of power penalty vs. normalized DGD for different GVD values to achieve a BER of $10^{-9}$ using exact pdf of the phase fluctuation due to GVD and PMD for 
4-QAM and PDM 4-QAM systems. For quantifying the power penalty, the power required for achieving the BER of $10^{-9}$ of 4-QAM system at zero GVD and zero DGD as the reference value. It is seen that the power penalty in PDM 4-QAM system is more than 4-QAM system. Moreover, the power penalty in PDM 4-QAM systems increases with the increase of DGD. Results show that for a BER of $10^{-9}$ at DGD of $0.5 \mathrm{~T}$ and GVD value of $1700 \mathrm{ps} / \mathrm{nm}$ the PDM 4-QAM system needs $2.21 \mathrm{~dB}$ more power than 4-QAM systems. It is also seen from this graph that the difference in power penalty increases with the increase in either GVD or DGD or both.

\section{Conclusion}

An analytical technique is presented to evaluate BER performance due to the combined influence of PMD and GVD of a coherent homodyne optical 4-QAM and PDM 4-QAM diversity transmission system using exact pdf of the random phase fluctuation. Results are evaluated for a $40 \mathrm{Gbps}$ optical 4-QAM and 80 Gbps PDM 4-QAM homodyne coherent systems with several values of system parameters. Results show that the system suffers significant power penalty due to combined influence of PMD and GVD at a given BER of $10^{-9}$. It is noticed that PDM 4-QAM system suffers more power penalty than the 4-QAM system. Further, the PDM 4-QAM system doubles the data rate. The PDM system provides a means of increasing the spectral efficiency at the cost of increased power penalty.

\section{References}

[1] K. A. Taher, and S. P. Majumder, "Analytical evaluation of the combined influence of PMD and GVD on the BER performance of optical homodyne QPSK systems", J. of Opt. Eng., 2017. vol. 56(12), pp. 126108-1-8.
[2] J. Yang, C. Yu et al., "CD-insensitive PMD monitoring based on RF power measurement", Opt. Express, 2011, vol. 19(2) pp. 1354-9.

[3] K. Murata et al., "100-Gbit/s PDM-QPSK coherent receiver with wide dynamic range and excellent common-mode rejection ratio," Opt. Express, 2011. vol. 29(26), pp. B125-130.

[4] C. Xie, "Impact of nonlinear and polarization effects in coherent systems," Opt. Express, vol. 19(26), 2011. pp. B915-30.

[5] Kesavan and H. Margaret, "Analysis of group velocity dispersion (GVD) with and without initial Gaussian chirp in different types of fiber," Int J. of Electrical, Computing Eng. and Commun. 2015, Vol. 1(1), pp. 1-6.

[6] V. K. Mishra, "Analytical approach to polarization mode dispersion in linearly spun fiber with birefringence," Int. J. of Optics, 2016, pp. 1-9.

[7] G. Edah et al., "Time-frequency approach to Gaussian and sinh-Gaussian pulse profiles propagating in a dispersive medium," African Review of Physics, 2015, pp. 47-53.

[8] R. Dar et al., "Properties of nonlinear noise in long, dispersion-uncompensated fiber links," Opt. Express, 2013, vol. 21(22), pp. 25685-25699.

[9] K. A. Taher and S. P. Majumder, "Analytical evaluation of the effect of cross polarization induced crosstalk on the BER performance of a PDM-QPSK coherent homodyne optical transmission system," J. of Optical Commun, 2016, pp. 1-10.

[10] K. A. Taher, S. P. Majumder, B. M. A. Rahman, Y. Yu and C. Yu, "Simultaneous monitoring of CD and PMD using RF tone power," Elsevier Procedia Eng., 2016, pp. 209-216. 